\title{
Three-N-butyphthalide alleviates early brain injury caused via subarachnoid hemorrhage via activating the LKB-1/ (AMP-activated protein kinase) pathway
}

\author{
XinNa ZHANG ${ }^{1}$, DongMei GUO ${ }^{1}$, Xin $Z_{H A N G}^{1}$, WenHui ZHANG ${ }^{1}$, Tao WANG ${ }^{1}$, Lei ZHANG ${ }^{1 *}$ (D)
}

\begin{abstract}
The influences of 3-N-butyphthalide (NBP) on early brain injury (EBI) induced via mild subarachnoid hemorrhage (SAH) and its mechanism were explored. Establishment of SAH model was via autologous blood injection. The protection of NBP on EBI in $\mathrm{SAH}$ model mice was evaluated. Determination of neuronal apoptosis was conducted. Detection of blood brain barrier (BBB), ZO-1, claudin-5, occludin and MMP-9 was manifested. The effects of NBP on oxidative stress, inflammation, and the SIRT1/ NF- $\kappa B$ and the LKB-1/AMP-activated protein kinase (AMPK) pathways were investigated. NBP could alleviate neurological deficits and $\mathrm{BBB}$ dysfunction, repress cerebral edema and mitigate brain histomorphology. NBP repressed apoptosis in brain tissues, oxidative stress, inflammation, NF- $\kappa B$ activity but facilitated SIRT1. Finally, NBP activated the LKB-1/AMPK pathway. NBP may lighten BBB dysfunction, oxidative stress, inflammation and apoptosis after SAH via activating the LKB-1/AMPK pathway, thereby mitigating EBI.
\end{abstract}

Keywords: butyphthalide; LKB-1/AMP-activated protein kinase; subarachnoid hemorrhage; early brain injury.

Pratical Application: 3-N-butyphthalide (NBP) on early brain injury (EBI) induced via mild subarachnoid hemorrhage.

\section{Introduction}

Subarachnoid hemorrhage (SAH) is a cerebrovascular illness with surprising mortality and disability rates. Although it only accounts for $5 \%$ of the occurrence of all stroke, the incidence in younger age, high mortality and disability rates, and unpleasing clinical influence result in a huge burden on the society (Pan et al., 2020). SAH is recognized as bleeding into the subarachnoid space, which is the area between the arachnoid membrane of the brain or spinal cord. Although a clear mitigation is manifested in minimally invasive interventions recently, the therapy outcomes for SAH patients remain unfortunate. Currently, more and more studies have clarified that early brain injury (EBI) within $72 \mathrm{~h}$ after the onset of $\mathrm{SAH}$ is regarded as the main reason of unpleasing prognosis of SAH patients (Fujii et al., 2013). EBI is defined as a suite of pathological processes bring about neuronal apoptosis and necrosis within $72 \mathrm{~h}$ after intracerebral hemorrhage. Current studies have confirmed that the pathological process of EBI consist of: 1. reduced cerebral blood flow caused via augmented intracranial pressure; 2 . damage of blood-brain barrier (BBB) and subsequent cerebral edema induced via multiple elements; 3. Brain inflammation stimulated via deficits in attention, motor control and perception pattern; 4. oxidative stress injury; 5. neuronal apoptosis and necrosis resulted from various factors eventually leading to serious damage to nerve function (Miller et al., 2014). Therefore, EBI therapy is considered to be the main aim of mitigating the prognosis of SAH patients.

Approval of 3-N-butyphthalide (NBP) was via China Food and Drug Administration for use in ischemic stroke in
2002 (Diao et al., 2015). In the meantime, NBP is therapeutic for a great many degenerative neurological diseases like Alzheimer's disease, dementia, amyotrophic lateral sclerosis, Parkinson's disease and other motor disorders (Chen et al., 2019). A mass of studies has pointed out that NBP can curb platelet aggregation, and oxidative damage caused by ischemia, modulate energy metabolism, lighten microcirculation and lessen the area of cerebral infarction. BBB dysfunction is crucial in EBI. Some studies have stated briefly that NBP can alleviate the BBB dysfunction caused via ischemia. For instance, NBP can perfect the BBB function via suppressing oxidative stress and activating Akt/GSK-3 $\beta / \beta$-catenin pathway in ischemia-hypoxic-induced brain injury (Ye et al., 2019). Furthermore, it has been pointed out that, in the model of local cerebral ischemia, NBP can relieve the experimental induced neurological defects after local cerebral hemorrhage via facilitating the formation of new blood vessels around the bleeding site (Tu et al., 2020).

The pharmacological mechanisms of NBP contains the protection of mitochondrial function, and repression of oxidative stress, inflammatory response and neuronal apoptosis. As BBB dysfunction, oxidative stress and inflammatory response are momentous in EBI, it can be assumed that NBP can alleviate SAH-induced EBI (Sun et al., 2013). Currently, few studies are conducted on the protective effect of NBP on EBI and its mechanism. This study was to probe into the effect of NBP on EBI stimulated via SAH and its mechanism. 


\section{Materials and methods}

\subsection{Breeding of experimental animals}

In this study, 60 adult male C57BL/6J mice (body weight between 20 and $25 \mathrm{~g}$ ) (Beijing Vital River Laboratory Animal Technology Co., Ltd., Beijing, China, Production license No.: SCXK (Jing) 2016-0006 or SCXK (Jing) 2016-0011) were raised in a pad box $(\mathrm{L} \times \mathrm{W} \times \mathrm{H}: 280 \mathrm{~mm} \times 160 \mathrm{~mm} \times 130 \mathrm{~mm})$ in a mouse breeding cage with 5 per cage in a specific pathogen-free grade environment with 12 -h alternating light.

\subsection{Animal model construction, neurological function score and brain edema degree measurement}

The construction approach of intravascular threading for $\mathrm{SAH}$ model is clarified in the literature, which is summarized as follows. Anesthesia of the mice was with isoflurane, then the left common carotid artery bifurcation was exposed to ligate and dissect the left external carotid artery. The nylon suture was inserted into the left internal carotid artery through the stump of the left external carotid artery and the bifurcation of the left common carotid artery. When the sutures were located near the anterior traffic artery, after the obstruction, the suture was pushed forward about $3 \mathrm{~mm}$. During and within $2 \mathrm{~h}$ after the operation, the temperature of the test animals was maintained at $36.5-37.5^{\circ} \mathrm{C}$ using heating pads. Postoperative analgesia was administered with meloxicam, and $5 \%$ glucose dissolved in normal saline was given as nutritional support. To guarantee the uniformity of injury, the mice with SAH grading score 7 or less were excluded.

At 24 or $72 \mathrm{~h}$ after SAH modeling, assessment of the neurological function of the mice was via the modified Garcia neurological score system. In short, the assessment comprised of the following six tests: spontaneous activity (0-3), limb movement symmetry (0-3), forelimb movement symmetry (0-3), climbing (0-3), response to trunk stimulation (0-3), and response to beard stimulation (0-3). The highest score was 18 and the lowest was 0 , with higher scores denoting better neurological function (Ferguson et al., 2008).

In this study, evaluation of the influence of NBP on cerebral edema was via cerebral water content indexes. The calculation method of cerebral water content was as follows: cerebral water content $(\%)=[($ wet - dry $) /$ wet $]$ weight $\times 100 \%$. Anesthesia and euthanasia of the mice 24 or $72 \mathrm{~h}$ after the operation were conducted. There were careful removing and assignation of the brain into four parts: left brain, right brain, cerebellum and brainstem. Then, detection of the water content was in each part separately. Weighing of the tissue was to gain wet weight, and the tissue was dried at $95 \sim 100{ }^{\circ} \mathrm{C}$ for $72 \mathrm{~h}$ and weighed again to obtain dry weight.

\subsection{Hematoxylin-Eosin (HE) staining}

Perfusion of the experimental mice was with phosphate buffer saline (PBS) $24 \mathrm{~h}$ after SAH modeling. After careful removing, fixation of the brain was in 4\% Paraformaldehyde (PFA) solution. Cut of the brain tissue was into sections of
$25 \mu \mathrm{M}$ thickness via Frozen section method. Conduction of HE staining was via a kit (BOSTER Biological Technology Co. Ltd., Wuhan, Hubei, China). Collection of images was via a microscope (LEICA DM500, USA).

\subsection{Fluoro-Jade C staining}

In this study, implementation of Fluoro-Jade C staining was to evaluate the influence of neuronal degeneration in NBP brain tissues. Perfusion of the experimental mice was with phosphate buffer saline (PBS) $72 \mathrm{~h}$ after SAH modeling. After careful removing, fixation of the brain was in $4 \%$ Paraformaldehyde (PFA) solution. Gradient dehydration was with 10\%, 20\%, and $30 \%$ sucrose solutions. Cut of the brain tissue was into sections of $25 \mu \mathrm{m}$ thickness via Frozen section method. There was incubation of the section with $1 \% \mathrm{NaOH}$ in $80 \%$ ethanol, watering with $70 \%$ ethanol, incubation with $0.06 \% \mathrm{KMnO} 4$ and $0.0001 \%$ FJC solution, and final microscopic examination.

\subsection{Integrity assessment of $B B B$}

In this study, application of Evans blue (EB) method was to assess the role of NBP on BBB integrity. Three h prior to euthanasia of the mice, injection of $4 \mathrm{~mL} / \mathrm{kg} \mathrm{2 \%}$ (w/v) EB solution was into the tail vein. After euthanasia of the mice, perfusion of $50 \mathrm{~mL}$ cold 0.1 M PBS solution was through the heart to remove the intravascular EB solution. At 24 and $72 \mathrm{~h}$ after SAH modeling, homogenization of brain tissues was with PBS and equal volume of trichloroacetic acid to precipitate protein. Then carrying out centrifugation was to extract the supernatant. Determination of the absorbance at $610 \mathrm{nM}$ was employed and that of $\mathrm{EB}$ extravasation was to evaluate the permeability of BBB.

\subsection{Western blot}

Twenty-four h after the SAH modeling, and prior to careful collection of the brain, ultrasonic lysis of the ipsilateral cortex samples of the mice was in a mixture of lysis buffer and $1 \%$ protease inhibitor, and then centrifugation was at $12,000 \mathrm{rpm} / \mathrm{min}$. Isolation of $20 \mu \mathrm{g}$ proteins was via sulfate polyacrylamide gel electrophoresis and electroblot was onto Polyvinylidene fluoride membranes (Millipore, USA) via wet transfer. Behind block with 5\% Bovine serum albumin solution, incubation was with the primary antibody and the secondary antibody combined with horseradish peroxidase. Finally, conduction of analysis was with the Bio-RAD imaging system (Bio-RAD, Hercules, CA, USA).

\subsection{TdT-mediated dUTP-biotin Nick End-Labeling (TUNEL) assay}

In this study, application of TUNEL commercial kit was to detect apoptosis in brain tissues. Perfusion of the mice was with PBS $24 \mathrm{~h}$ after SAH modeling. After careful removing, fixation of the brain was in $4 \%$ PFA solution and gradient dehydration was with $10 \%, 20 \%$, and $30 \%$ sucrose solutions. Cut of the brain tissue was into sections of $25 \mu \mathrm{m}$ thickness via Frozen section method. Incubation of the section was with $0.3 \%$ hydrogen peroxide, TUNEL reaction solution, 4', 6-diamidino-2-phenylindole $(5 \mu \mathrm{g} / \mathrm{mL})$ solution, followed by microscopic examination. 


\subsection{Determination of oxidative stress indexes}

Twenty-four h after SAH modeling, taking of the brain of the mice was after PBS perfusion. Detection of malondialdehyde (MDA), superoxide dismutase (SOD), glutathione peroxidase (GSH-Px) and Catalase (CAT) was in cerebral cortex. On the grounds of the instructions of the commercial kit, adhibition of the thibabituric acid method was to make MDA react with thiobarbituric acid, and then measurement of the absorbance and determination of MDA were put into use. Detection of SOD activity was via WST-1 method on the basis of the instructions of commercial kits. In the meantime, determination of GSH-Px activity was also submitted to instructions of commercial kits. Moreover, examination of the reducibility of NADPH was in the reaction system.

Adhibition of Enzyme-linked immunosorbent assay (ELISA) kits was to evaluate 3-nitrotyrosine (3-NT), 8-oxo-deoxyguanosine (8-OHdG) and CAT in injured tissues $24 \mathrm{~h}$ after SAH. In short, incubation of the sample or standard substances was with primary antibody, secondary antibody and substrate solution. Finally, addition of the solution stopping the reaction was manifested. Measurement of the absorbance was via microplate reader (Molecular Devices, Sunnyvale, CA, USA) and calculation of 3-NT, 8-OHdG, and CAT was clarified.

\subsection{Statistical experimental methods}

In this study, expression of all quantitative indexes was in the form of calculating mean \pm standard deviation. Levene's application was to test homogeneity of variance. If no statistical significance was manifested $(\mathrm{P}>0.05)$, adhibition of one-way analysis of variance (ANOVA) was for statistical analysis. If
ANOVA was statistically significant $(\mathrm{P} \leq 0.05)$, application of LSD was for a comprehensive comparison. If the variance was not uniform ( $\mathrm{P} \leq 0.05)$, Kruskal-Wallis test was conducted. If the Kruskal-Wallis test was statistically significant $(\mathrm{P} \leq 0.05)$, adhibition of Mann-Whitney method was for pair comparison in mean.

\section{Results}

\subsection{Influences of NBP on neurological function, brain edema, brain histomorphology and neuronal degeneration after $S A H$}

For verification of the success of SAH modeling and evaluation of the influences of NBP on the cerebral water content and neurological deficit score stimulated via $\mathrm{SAH}$, measurement of the neurological deficit score was of each group $24 \mathrm{~h}$ after $\mathrm{SAH}$. The results manifested that the neurological score was apparently declined in the SAH versus the sham, while clearly elevated in the comparison of the SAH with the $\mathrm{NBP}+\mathrm{SAH}$ $(\mathrm{P}<0.05$, Figure $1 \mathrm{~A})$.

Additionally, versus the sham, apparent augmented water content of different anatomical brain structures (left brain, right brain, cerebellum and brainstem) was in the SAH at 24 and $72 \mathrm{~h}(\mathrm{P}<0.05)$, with distinct cutting down water content in the brain tissues in the SAH + NBP treatment $(\mathrm{P}<0.05)$ (Figure 1B). Subsequently, at $72 \mathrm{~h}$, assessment of the difference in brain histomatology of the mice in each group clarified clearly mitigating brain histomatology of the SAH + NBP versus the SAH (Figure 1C).
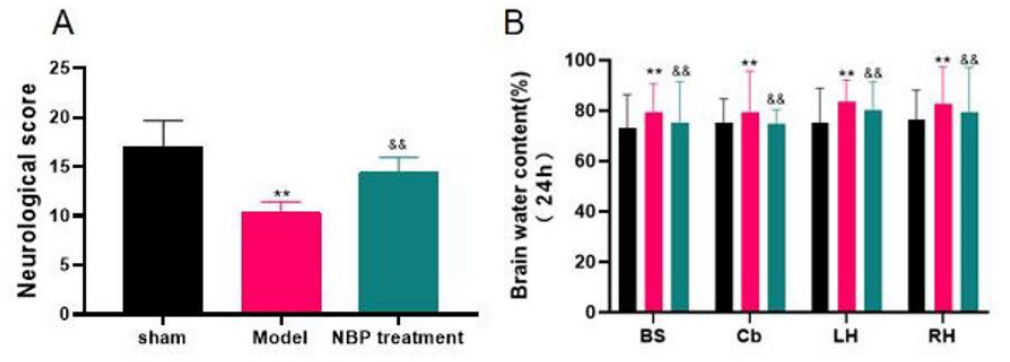

C

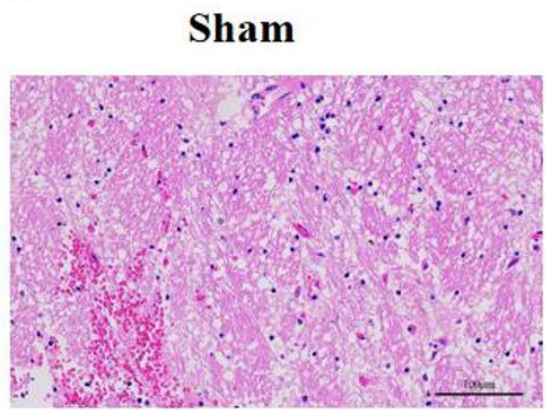

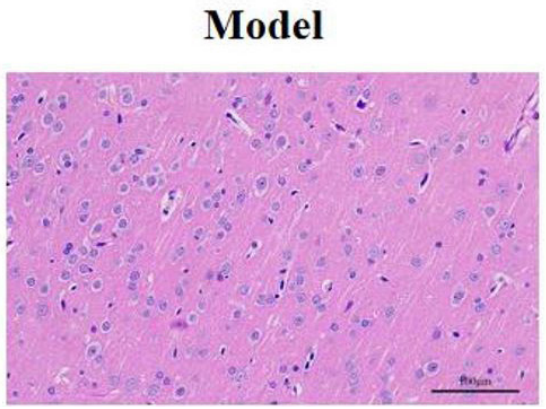

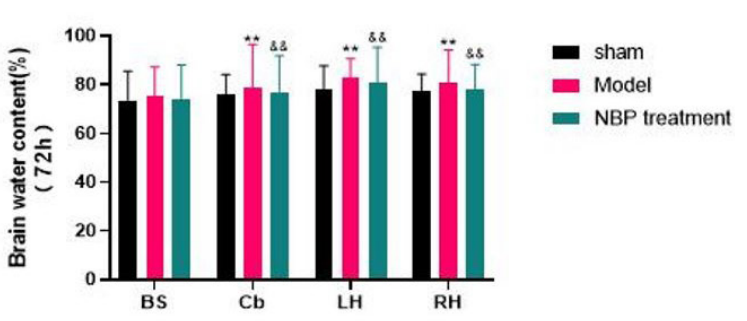

Figure 1. The impacts of NBP on neurological function, cerebral edema, cerebral histomorphology and neuronal degeneration after SAH. (A) NBP treatment distinctly mitigated the neurological function of SAH mice $(\mathrm{n}=12) ;(\mathrm{B})$ NBP brought down cerebral water content within 24 and $72 \mathrm{~h}$ after SAH; (C) HE staining clarified that NBP alleviated the brain histomorphology $(\mathrm{n}=3)$. BS represented brainstem, Cb for cerebellum, LH for left brain, RH for right brain; ${ }^{* *}$, the SAH vs the sham, $\mathrm{P}<0.05 ; \& \&$, the SAH + NBP vs the SAH, $\mathrm{P}<0.05$. 


\subsection{NBP protects SAH-induced BBB integrity}

SAH-induced BBB dysfunction is a crucial pathological phenomenon in EBI. In this study, to evaluate the impact of NBP on SAH-induced BBB integrity, examination of the consequence of different treatments on EB extravasation at 24 and $72 \mathrm{~h}$ after SAH injury affirmed that remarkable ascent of EB content was in brain tissues of the mice in the SAH versus the sham, and obvious descending EB content was manifested in a contrast of the SAH and the SAH + NBP (Figure 2A).

For further exploration of the role of NBP on molecular indicators linked with $\mathrm{BBB}$ integrity in SAH model at the molecular level, the impacts of NBP on key molecules in BBB dysfunction (ZO-1, MMP-9, claudin-5, occludin) were discussed. The results clarified markedly rising MMP-9 and dramatical cutting down ZO-1, claudin-5, occludin in a contrast of the SAH with the sham in the brain tissues of the mice, which was adverse in a comparison of the SAH and the SAH + NBP (Figure 2B). Shortly, $\mathrm{NBP}$ protected $\mathrm{SAH}$-induced $\mathrm{BBB}$ integrity.

\subsection{NBP represses neuronal apoptosis in cerebral cortex stimulated via SAH}

In this study, for exploration of the role of NBP on SAHinduced apoptosis in brain tissues, measurement of the apoptosis of cortical neurons was in each group of the mice. As clarified in Figure 3A, clear augmented number of apoptotic cells was manifested in the $\mathrm{SAH}$ versus the sham, which was opposite in the NBP $+\mathrm{SAH}$ at $24 \mathrm{~h}$ time point $(\mathrm{P}<0.05)$.

For further exploration of the possible mechanism of NBP curbing cerebral cortex apoptosis stimulated via $\mathrm{SAH}$, detection of the impacts of NBP was on Bax, Bcl-2, Cyt C and active caspase 9, clarifying that NBP was available to obviously repress Bax, Cyt $C$ and active-caspase 9 but accelerate $B c l-2$ versus the $\mathrm{SAH}$ (Figure 3B). All in all, NBP could memorably curb the apoptosis of cerebral cortex neurons caused via SAH.

\subsection{NBP constrains SAH-induced inflammation and the SIRT1/NF-kB pathway}

Brain inflammation is momentous in the EBI of SAH. To determine the role of NBP on neuroinflammation, detection of inflammatory cytokines IL- 6 , IL- $1 \beta$, TNF- $\alpha$ and IL- 10 was in rat brain tissues $72 \mathrm{~h}$ after $\mathrm{SAH}$, testifying the clear augmented pro-inflammatory cytokines IL-6, IL-1 $\beta$, TNF- $\alpha$, and dramatically descending anti-inflammatory cytokine IL-10 in the SAH versus the sham, which was contrast after NBP addition $(\mathrm{P}<0.05$, Figure 4B).

For further exploration of the molecular mechanism of NBP restraining $\mathrm{SAH}$-induced brain inflammation, determination of the impacts of NBP was on the SIRT1/NF- $\kappa B$ pathway, which is crucial in SAH-induced brain inflammation. The results clarified degressive SIRT1 in brain tissues of the mice, and ascending intranuclear NF- $\mathrm{kB}$ in the SAH versus the sham, which was opposite in a contrast of the SAH with the NBP + SAH $(\mathrm{P}<0.05$, Figure $4 \mathrm{~A})$.

\subsection{NBP curbs SAH-induced oxidative stress}

Nowadays, numerous studies have clarified that oxidative stress is vital in the pathological mechanism of EBI stimulated via SAH. First, it was affirmed remarkably augmented gp91phox
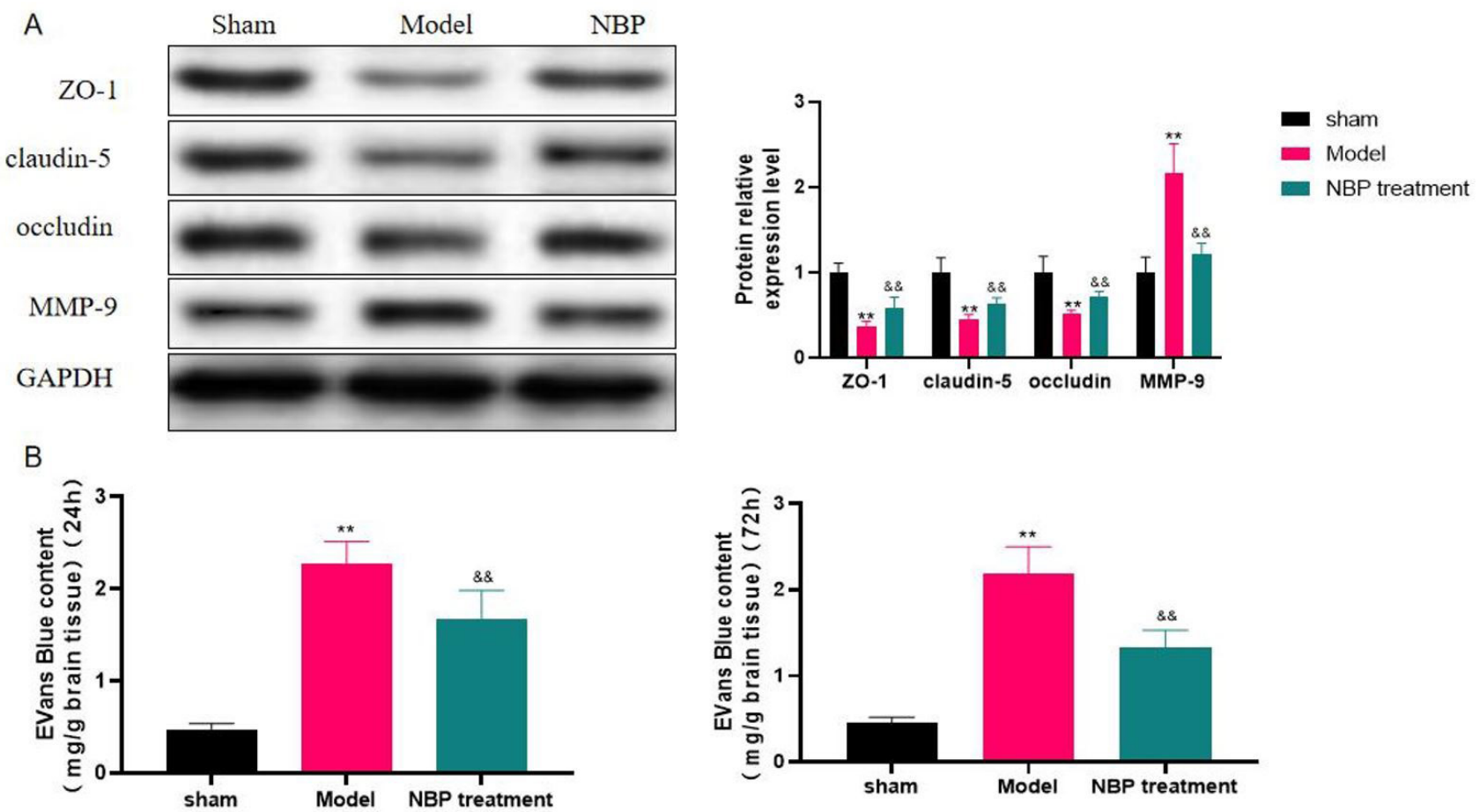

Figure 2. NBP protects BBB integrity. (A) The impacts of NBP on the amount of EB exosmosis at 24 and $72 \mathrm{~h}(\mathrm{n}=6)$; (B) Western blot to detect the impacts of NBP on ZO-1, claudin-5, occludin and MMP-9 $(n=3){ }^{* *}$, the SAH vs the sham, $\mathrm{P}<0.05$; \& \&, the SAH + NBP vs the SAH, P $<0.05$. 
A Sham

Model NBP
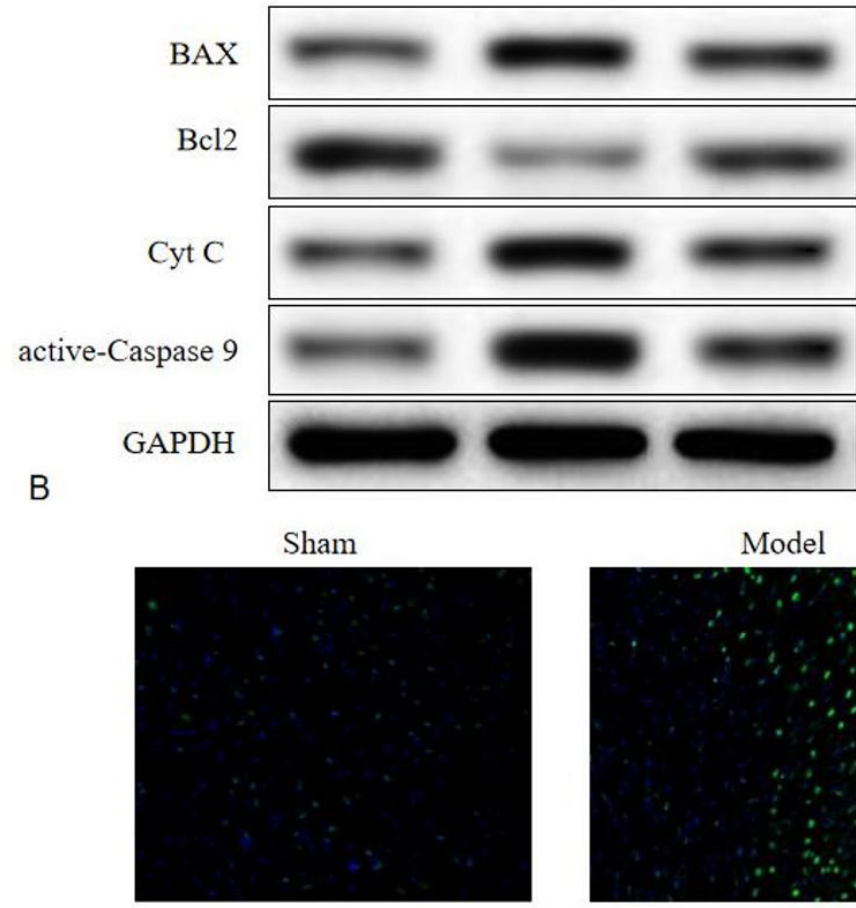
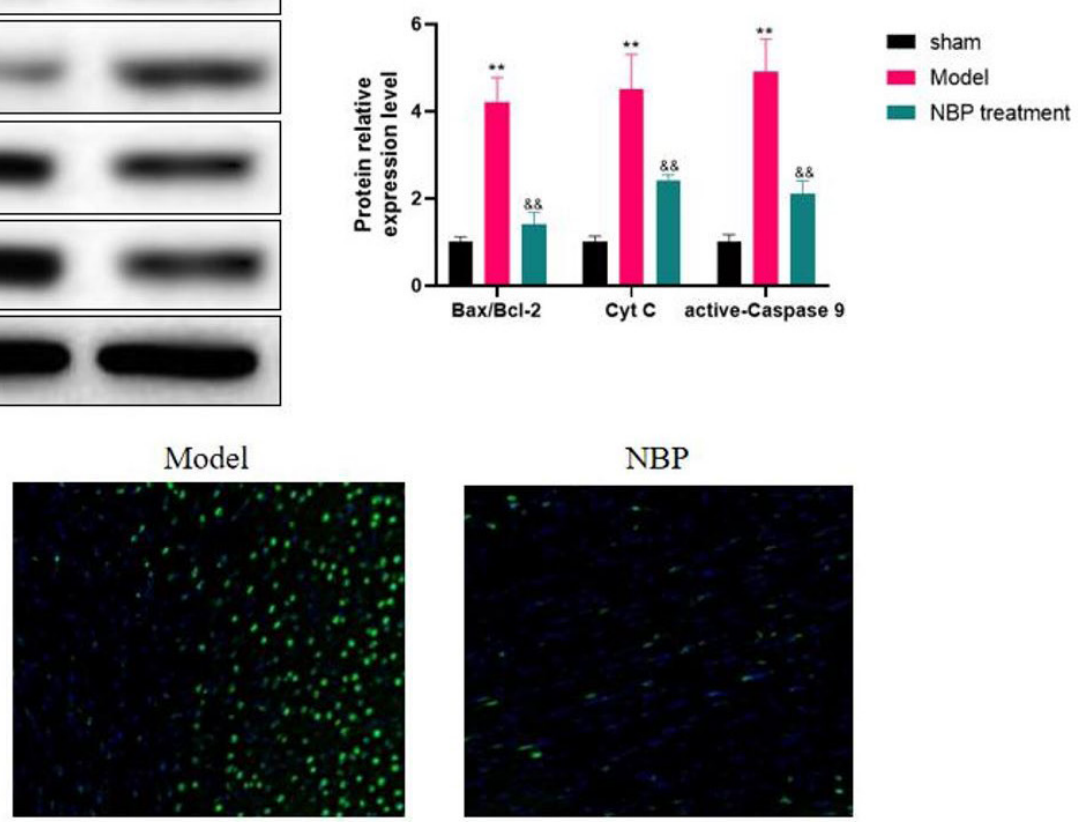

Figure 3. NBP restrains neuronal apoptosis of cerebral cortex caused via SAH. (A) TUNEL detection of NBP on SAH-induced neuronal apoptosis in mouse cerebral cortex; (B) Western blot to examine the impacts of NBP on apoptosis-linked proteins Bax, Bcl-2, Cyt C and active-caspase 9. **, the SAH vs the sham, $\mathrm{P}<0.05 ; \& \&$, the $\mathrm{SAH}+\mathrm{NBP}$ vs the $\mathrm{SAH}, \mathrm{P}<0.05$.

A

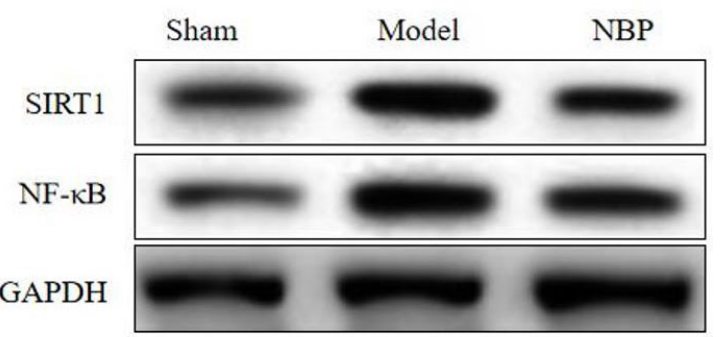

B
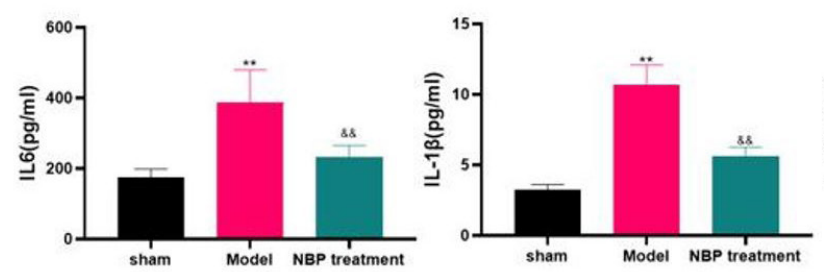

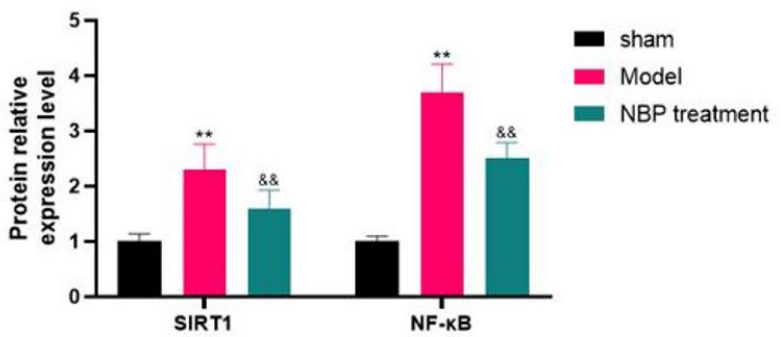

NBP

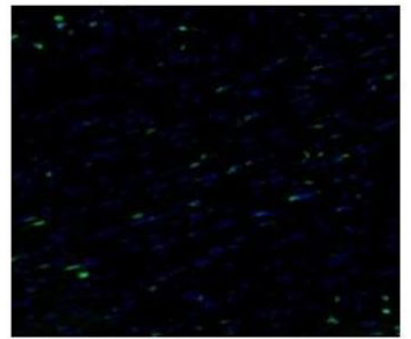

Figure 4. NBP depresses SAH-induced inflammation and the SIRT1/NF- $\kappa B$ pathway. (A) Western blot analysis of the impacts of NBP on the SIRT1/NF- $\mathrm{kB}$ pathway $(\mathrm{n}=3)$; (B) ELISA to examine the impacts of NBP on neuroinflammation stimulated via SAH model $72 \mathrm{~h}$ after SAH modeling $(n=6)$. ${ }^{* *}$, the SAH vs the sham, $\mathrm{P}<0.05 ; \& \&$, the $\mathrm{SAH}+\mathrm{NBP}$ vs the $\mathrm{SAH}, \mathrm{P}<0.05$.

and 4-HNE in cerebral cortex of the mice in the SAH $24 \mathrm{~h}$ after SAH modeling, which was prominently refrained via NBP $(\mathrm{P}<0.05$, Figure $5 \mathrm{~A})$.

Detection results clarified remarkable augment of MDA, 8-OHdG and 3-NT, and descent of SOD, GSH-Px and CAT in the brain tissue of the mice $24 \mathrm{~h}$ after $\mathrm{SAH}$, but the indexes were changed via NBP addition $(P<0.05$, Figure 5B, C).

\subsection{NBP activates the LKB-1/AMP-Activated Protein Kinase (AMPK) pathway}

The activation of LKB-1/AMPK pathway is momentous in intracellular biological processes like mitochondrial function, cell growth, glucose and lipid metabolism, oxidative stress, and maintenance of mitochondrial homeostasis. In the meantime, it has been affirmed that activation of LKB-1/AMPK pathway 
A

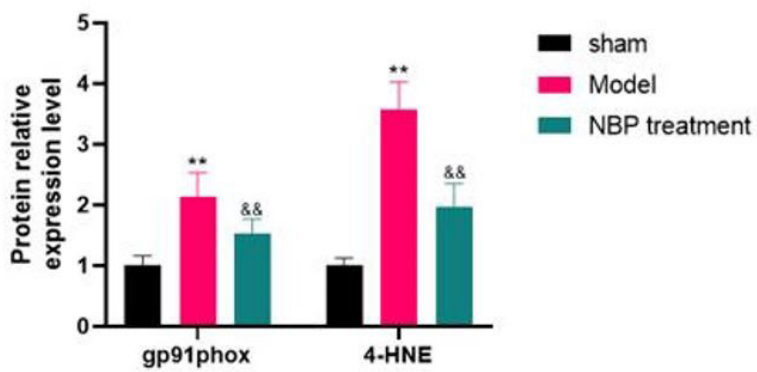

B
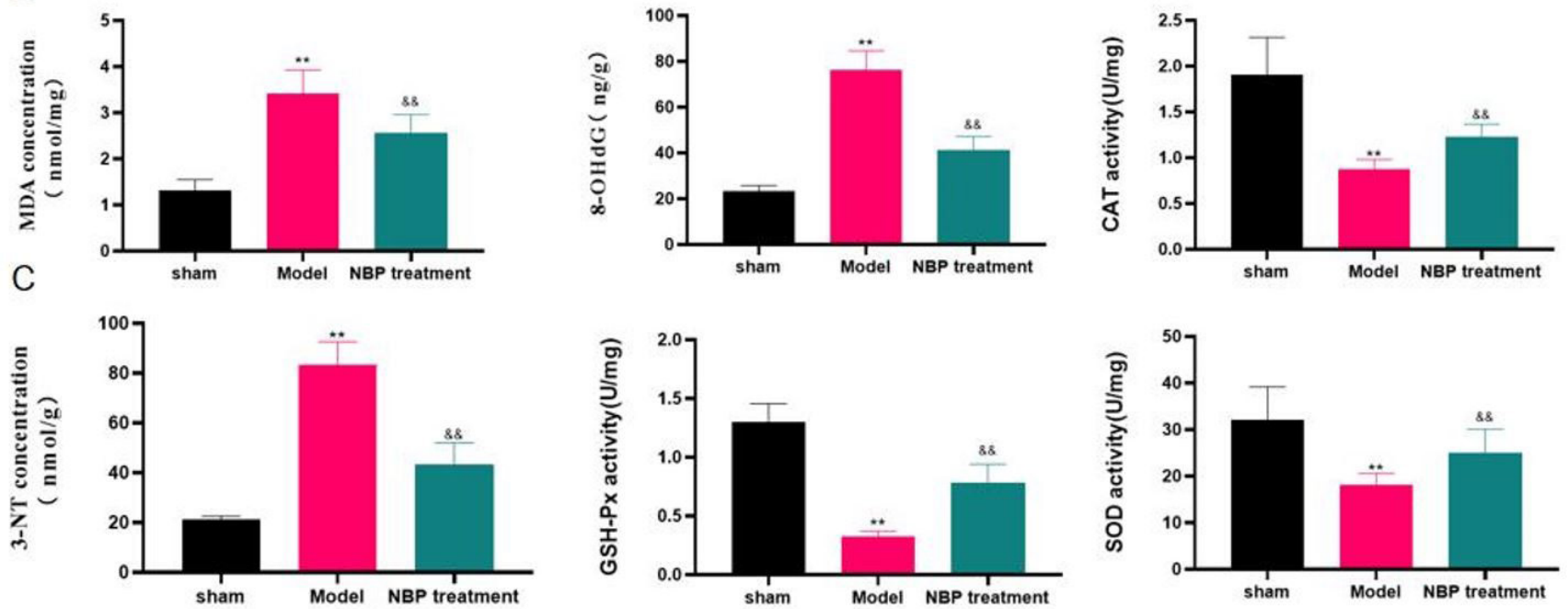

Figure 5. NBP curbs SAH-induced oxidative stress. (A) Western blot analysis of the impacts of NBP on gp91phox and 4-HNE ( $\mathrm{n}=6$ ); (B) The impacts of NBP on MDA, 8-OHdG and 3-NT in rat brain tissues detected via ELISA $(n=6)$; (C) The impacts of NBP on SOD, GSH-Px and CAT contents detected via ELISA $(\mathrm{n}=3)$. ${ }^{* *}$, the $\mathrm{SAH}$ vs the sham, $\mathrm{P}<0.05 ; \& \&$, the SAH + NBP vs the SAH, $\mathrm{P}<0.05$.
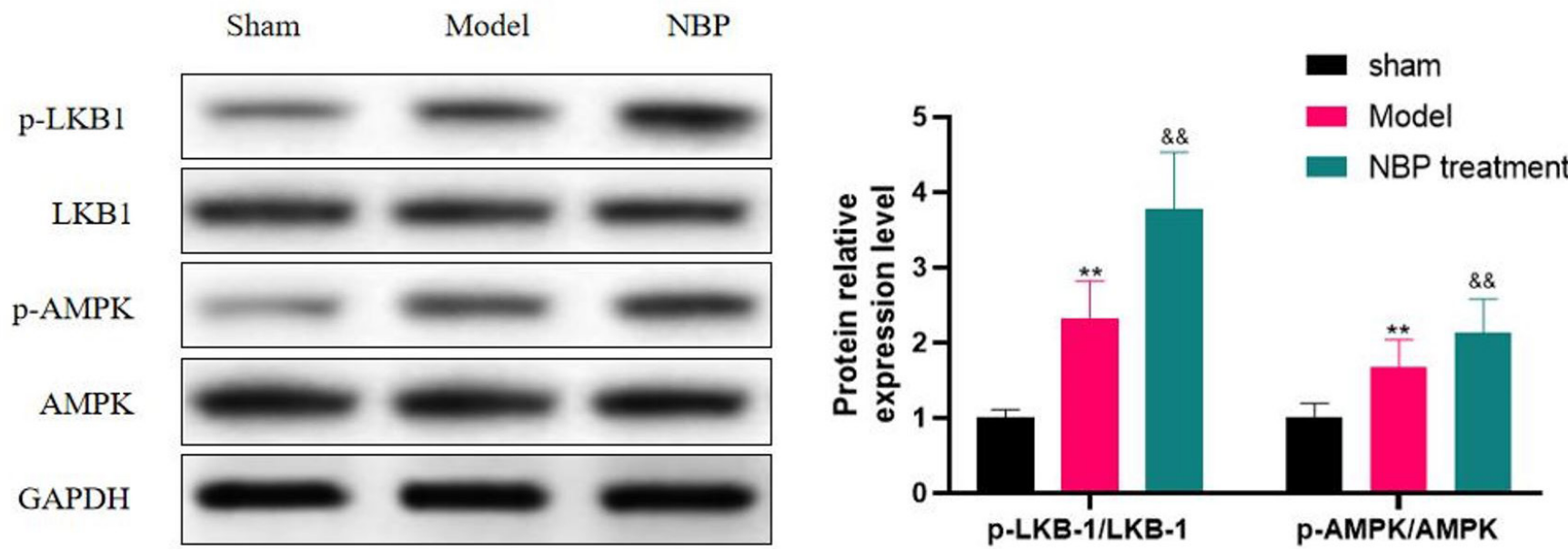

Figure 6. NBP enables the LKB-1/AMPK pathway. Western blot to examine the impacts of NBP on p-LKB-1/LKB-1 and p-AMPK/AMPK ( $\mathrm{n}=3$ ). **, the $\mathrm{SAH}$ vs the sham, $\mathrm{P}<0.05 ; \& \&$, the $\mathrm{SAH}+\mathrm{NBP}$ vs the $\mathrm{SAH}, \mathrm{P}<0.05$.

is available to curb SAH model-induced EBI. In the study, for further discussion of the mechanism of NBP refraining SAHinduced EBI, detection of the impacts of NBP was on the LKB-1/ AMPK pathway.

As clarified in Figure 6, remarkable augment of p-LKB-1/ LKB-1 and p-AMPK/AMPK was manifested after SAH versus the sham, which was strengthened via NBP treatment $(\mathrm{P}<0.05)$.
Briefly, NBP was available to memorably activate the LKB-1/ AMPK pathway, which was implicated with the protection of NBP on SAH-induced EBI.

\section{Discussion}

SAH is a cerebrovascular illness with surprising mortality and disability rates. Although occupying $5 \%$ of the incidence of 
all stroke, owing to accompanied cognitive impairment in SAH patients, the illness is still seriously influential in their ability to work and quality of life, resulting in a huge burden to the society. Nevertheless, with some advancement in the diagnosis, treatment and surgical methods of SAH, the prognosis of SAH is still not ideal (Chen et al., 2014). For instance, a current study affirms that the death and disability rate of SAH is up to $45 \%$. Therefore, the search for better treatments is greatly urgent. EBI is a vital reason of disability and death after SAH. EBI-targeted therapy is available to successfully refrain secondary injury and alleviate the prognosis of SAH patients (Ansar et al., 2011). In consequence, targeting EBI to restrain EBI injury is a crucial method to cut down the disability rate and mortality rate of SAH patients.

NBP, also named as race-3-n-NBP, is a type of novel drugs independently developed in China. Vast studies have affirmed that NBP manifests some pharmacological impacts, like suppression of anti-platelet aggregation, neuronal apoptosis and neuroinflammation, mitigation of microcirculation and energy metabolism, prevention of thrombosis, protection of mitochondria, and fighting free radicals (Chaudhry et al., 2018). In this study, it was assured that: 1 . NBP was available to obviously curb the neurological deficits stimulated via SAH model, 2. BBB dysfunction and brain edema, mitigate brain histomorphology; 3. NBP refrained apoptosis in brain tissues, oxidative stress, NF- $\kappa \mathrm{B}$ activity and neuroinflammation but accelerated SIRT1; 4. NBP motivated the LKB-1/AMPK pathway. All in all, NBP may mitigate EBI via enabling the LKB-1/AMPK pathway, repressing $\mathrm{BBB}$ dysfunction, oxidative stress, inflammation, and apoptosis after $\mathrm{SAH}$.

SAH-stimulated apoptosis, oxidative stress, inflammation and $\mathrm{BBB}$ dysfunction are momentous in the pathophysiological changes of EBI (Zolnourian et al., 2019). For instance, BBB dysfunction resulted from a series of inflammatory factors, oxidative stress and augmented MMP9 directly leads to cytotoxic cerebral edema, ultimately giving rise to positive feedback brain injury (Zhu et al., 2018). Tight intercellular contact between microvascular endothelial cells constitutes the functional structure of BBB, in which ZO-1, claudin-5 and occludin are essential(Ramirez et al., 2013). In this study, NBP remarkably refrained $\mathrm{SAH}$-induced $\mathrm{BBB}$ dysfunction and subsequent cerebral edema, strengthened ZO-1, claudin-5 and occludin, and curbed MMP9 participating in the decomposition of extracellular matrix.

Apoptosis, oxidative stress injury and inflammation caused via Reactive oxygen species (ROS) excess are the kernel of pathological processes of EBI. Meanwhile, ROS' augment in $\mathrm{EBI}$ is able to give rise to severe brain tissue damage (Liu et al., 2020). In this study, NBP was available to markedly refrain MDA, 8-OHdG and 3-NT in brain tissues. MDA, 8-OHdG and 3 -NT are outcomes of the chain reaction of lipid peroxidation, and their levels can manifest the level of lipid peroxidation in brain tissues. In the meantime, NBP could memorably constrain gp91phox and 8-OHdG in brain tissues. Eight-OHdG, a specific marker of DNA damage, is frequently applied to evaluate the degree of DNA damage resulted from ROS. As another marker of oxidative stress, gp91phox, affiliated from the NADPH oxidase family, is available to produce ROS (Wang et al., 2017).
Simultaneously, it was affirmed that NBP could obviously upregulate SOD, GSH-Px and CAT. SOD, a crucial antioxidant, is capable of converting superoxide anion into oxygen and hydrogen peroxide (Baskoylu et al., 2018). GSH-Px has noteworthy antioxidative stress impacts, can protect the structure and function of cell membrane from the interference and injure of oxide . CAT is available to decompose hydrogen peroxide into water and oxygen, and is the central enzyme of antioxidant stress response (Sousa et al., 2019).

Neuronal apoptosis is essential in the pathogenesis of EBI. $\mathrm{Bcl}-2$ and Bax are crucial in modulating apoptosis. Additionally, Bax is beneficial for activation of Caspase pathway. Bcl-2 is available to repress the activity of Bax whose elevation accelerates apoptosis (Maes et al., 2017). In this study, NBP remarkably depressed the elevation of BAX, Cyt C, and active Caspase 9 stimulated via $\mathrm{SAH}$, and facilitated anti-apoptotic protein $\mathrm{Bcl}-2$. Moreover, NBP memorably restrained the apoptosis stimulated via $\mathrm{SAH}$, refraining the neurological dysfunction after SAH.

Current animal researches evidences clarify that SAHinduced neuroinflammation is essential in the pathophysiological mechanism of EBI (Wang et al., 2018). For instance, strengthening inflammatory cytokines IL-6, IL- $1 \beta$ and TNF- $\alpha$ are manifested in cerebrospinal fluid of SAH patients. The research has pointed out that inflammatory activation is implicated with cell damage stimulated via $\mathrm{SAH}$, and targeting inflammation is available to memorably alleviate the prognosis of SAH patients, in favor of the treatment of SAH. In this study, NBP could curb SAH-induced elevated IL- 6 , IL- $1 \beta$ and TNF- $\alpha$, but enhanced SIRT1. SIRT1, a part of the sirtuins family, is available to control apoptosis, inflammation and other crucial biological processes (Hwang et al., 2013). Numerous studies have clarified that motivation of SIRT1 can lighten SAH-induced neuronal apoptosis and neuroinflammation in animal models of SAH, with expediting ubiquitination and degradation of $\mathrm{NF}-\kappa \mathrm{B}, \mathrm{P} 53$ and FOXO3. The findings clarified that NBP could motivate SIRT1 and refrain $\mathrm{NF}-\kappa \mathrm{B}$, thus exerting anti-inflammatory influence. AMPK, an extremely conserved sensor of cellular energy status and a main modulator of eukaryotic cell responses to restrained ATP (Hardie, 2018). AMPK can be abnormally motivated via phosphorylation of AMPK's upstream signaling molecule LKB1 and stimulation of pathological manifestations like oxidative damage, hypoxia and glucose deficiency (Venna et al., 2012; Eom et al., 2016). Therefore, phosphorylation of AMPK on its downstream target causes motivation of catabolic pathway of ATP production and depression of ATP consumption. In this study, NBP remarkably expedited the LKB-1/AMPK pathway. A good deal of studies has clarified that AMPK can apparently depress neuronal apoptosis stimulated via ischemia and hypoxia. For instance, pretreatment of Apelin-13 could motivate AMPK phosphorylation and refrain apoptosis after cerebral ischemia, which is mitigated via addition of AMPK depressor Compound C (Duan et al., 2019). In the meantime, AMPK agonists is available to motivate AMPK pathway to refrain neuroinflammation damage to the central nervous system (Jiang et al., 2018). For instance, metformin facilitates phosphorylation of AMPK and restrains neutrophil infiltration to constrain NF- $\kappa \mathrm{B}$, IL-1 $\beta$, IL-6, and TNF- $\alpha$ in MACO models. Moreover, a study in vitro affirms that metformin could restrain the augment of ICAM-1 stimulated via 
OGD model through the motivated AMPK pathway, which was reversed via Compound C. All in all, AMPK could memorably curb neuroinflammation. Furthermore, NBP distinctly motivated the SIRT1/NF- $\mathrm{kB}$ pathway, which was supposed to be implicated with motivated AMPK pathway, which is an upstream molecule of SIRT1 and available to up-regulate SIRT1 (Li et al., 2017).

In brief, in this study it was confirmed the protective impact of NBP on EBI in SAH mice. NBP is supposed to restrain BBB injury, oxidative stress, neuroinflammation and apoptosis via motivative LKB-1/AMPK pathway. The powerful protective impact of NBP on EBI after SAH presumably make it a promising treatment for SAH.

\section{References}

Ansar, S., Maddahi, A., \& Edvinsson, L. (2011). Inhibition of cerebrovascular raf activation attenuates cerebral blood flow and prevents upregulation of contractile receptors after subarachnoid hemorrhage. BMC Neuroscience, 12(1), 107. http://dx.doi.org/10.1186/1471-2202-12107. PMid:22032648.

Baskoylu, S. N., Yersak, J., O’Hern, P., Grosser, S., Simon, J., Kim, S., Schuch, K., Dimitriadi, M., Yanagi, K. S., Lins, J., \& Hart, A. C. (2018). Single copy/knock-in models of ALS SOD1 in C. elegans suggest loss and gain of function have different contributions to cholinergic and glutamatergic neurodegeneration. PLOS Genetics, 14(10), e1007682. http://dx.doi.org/10.1371/journal.pgen.1007682. PMid:30296255.

Chaudhry, S. R., Hafez, A., Jahromi, B. R., Kinfe, T. M., Lamprecht, A., Niemelä, M., \& Muhammad, S. (2018). Role of Damage Associated Molecular Pattern Molecules (DAMPs) in Aneurysmal Subarachnoid Hemorrhage (aSAH). International Journal of Molecular Sciences, 19(7), 2035. http://dx.doi.org/10.3390/ijms19072035. PMid:30011792.

Chen, S., Feng, H., Sherchan, P., Klebe, D., Zhao, G., Sun, X., Zhang, J., Tang, J., \& Zhang, J. H. (2014). Controversies and evolving new mechanisms in subarachnoid hemorrhage. Progress in Neurobiology, 115, 64-91. http://dx.doi.org/10.1016/j.pneurobio.2013.09.002. PMid:24076160.

Chen, X. Q., Qiu, K., Liu, H., He, Q., Bai, J. H., \& Lu, W. (2019). Application and prospects of butylphthalide for the treatment of neurologic diseases. Chinese Medical Journal, 132(12), 1467-1477. http://dx.doi.org/10.1097/CM9.0000000000000289. PMid:31205106.

Diao, X. X., Zhong, K., Li, X. L., Zhong, D. F., \& Chen, X. Y. (2015). Isomer-selective distribution of 3-n-butylphthalide (NBP) hydroxylated metabolites, 3-hydroxy-NBP and 10-hydroxy-NBP, across the rat blood-brain barrier. Acta Pharmacologica Sinica, 36(12), 1520-1527. http://dx.doi.org/10.1038/aps.2015.64. PMid:26567730.

Duan, J., Cui, J., Yang, Z., Guo, C., Cao, J., Xi, M., Weng, Y., Yin, Y., Wang, Y., Wei, G., Qiao, B., \& Wen, A. (2019). Neuroprotective effect of Apelin 13 on ischemic stroke by activating AMPK/GSK-3 $\beta / \mathrm{Nrf} 2$ signaling. Journal of Neuroinflammation, 16(1), 24. http://dx.doi. org/10.1186/s12974-019-1406-7. PMid:30709405.

Eom, J. W., Lee, J. M., Koh, J. Y., \& Kim, Y. H. (2016). AMP-activated protein kinase contributes to zinc-induced neuronal death via activation by LKB1 and induction of Bim in mouse cortical cultures. Molecular Brain, 9(1), 14. http://dx.doi.org/10.1186/s13041-0160194-6. PMid:26856538.

Ferguson, M., Rebrin, I., Forster, M. J., \& Sohal, R. S. (2008). Comparison of metabolic rate and oxidative stress between two different strains of mice with varying response to caloric restriction.
Experimental Gerontology, 43(8), 757-763. http://dx.doi.org/10.1016/j. exger.2008.04.016. PMid:18541398.

Fujii, M., Yan, J., Rolland, W. B., Soejima, Y., Caner, B., \& Zhang, J. H. (2013). Early brain injury, an evolving frontier in subarachnoid hemorrhage research. Translational Stroke Research, 4(4), 432-446. http://dx.doi.org/10.1007/s12975-013-0257-2. PMid:23894255.

Hardie, D. G. (2018). Keeping the home fires burning: AMP-activated protein kinase. Journal of the Royal Society, Interface, 15(138), 20170774. http://dx.doi.org/10.1098/rsif.2017.0774. PMid:29343628.

Hwang, J. W., Yao, H., Caito, S., Sundar, I. K., \& Rahman, I. (2013). Redox regulation of SIRT1 in inflammation and cellular senescence. Free Radical Biology \& Medicine, 61, 95-110. http://dx.doi.org/10.1016/j. freeradbiomed.2013.03.015. PMid:23542362.

Jiang, S., Li, T., Ji, T., Yi, W., Yang, Z., Wang, S., Yang, Y., \& Gu, C. (2018). AMPK: potential therapeutic target for ischemic stroke. Theranostics, 8(16), 4535-4551. http://dx.doi.org/10.7150/thno.25674. PMid:30214637.

Li, X., Lee, Y. J., Jin, F., Park, Y. N., Deng, Y., Kang, Y., Yang, J. H., Chang, J. H., Kim, D. Y., Kim, J. A., Chang, Y. C., Ko, H. J., Kim, C. H., Murakami, M., \& Chang, H. W. (2017). Sirt1 negatively regulates FceRI-mediated mast cell activation through AMPK- and PTP1Bdependent processes. Scientific Reports, 7(1), 6444. http://dx.doi. org/10.1038/s41598-017-06835-3. PMid:28744004.

Liu, H., Guo, W., Guo, H., Zhao, L., Yue, L., Li, X., Feng, D., Luo, J., Wu, X., Cui, W., \& Qu, Y. (2020). Bakuchiol attenuates oxidative stress and neuron damage by regulating Trx1/TXNIP and the phosphorylation of AMPK after subarachnoid hemorrhage in mice. Frontiers in Pharmacology, 11, 712. http://dx.doi.org/10.3389/ fphar.2020.00712. PMid:32499702.

Maes, M. E., Schlamp, C. L., \& Nickells, R. W. (2017). BAX to basics: how the BCL2 gene family controls the death of retinal ganglion cells. Progress in Retinal and Eye Research, 57, 1-25. http://dx.doi. org/10.1016/j.preteyeres.2017.01.002. PMid:28064040.

Miller, B. A., Turan, N., Chau, M., \& Pradilla, G. (2014). Inflammation, vasospasm, and brain injury after subarachnoid hemorrhage. BioMed Research International, 2014, 384342. http://dx.doi. org/10.1155/2014/384342. PMid:25105123.

Pan, C. Y., Tian, M., Zhang, L. L., Tian, D., Wang, L. Y., Sun, Y. J., \& Cui, Y. F. (2020). lncRNA signature for predicting cerebral vasospasm in patients with SAH: implications for precision neurosurgery. Molecular Therapy. Nucleic Acids, 21, 983-990. http://dx.doi.org/10.1016/j. omtn.2020.07.028. PMid:32818922.

Ramirez, S. H., Fan, S., Dykstra, H., Rom, S., Mercer, A., Reichenbach, N. L., Gofman, L., \& Persidsky, Y. (2013). Inhibition of glycogen synthase kinase $3 \beta$ promotes tight junction stability in brain endothelial cells by half-life extension of occludin and claudin-5. PLoS One, 8(2), e55972. http://dx.doi.org/10.1371/journal.pone.0055972. PMid:23418486.

Sousa, R. H. V., Carvalho, F. E. L., Lima-Melo, Y., Alencar, V., Daloso, D. M., Margis-Pinheiro, M., Komatsu, S., \& Silveira, J. A. G. (2019). Impairment of peroxisomal APX and CAT activities increases protection of photosynthesis under oxidative stress. Journal of Experimental Botany, 70(2), 627-639. http://dx.doi.org/10.1093/ jxb/ery354. PMid:30312463.

Sun, X., Ji, C., Hu, T., Wang, Z., \& Chen, G. (2013). Tamoxifen as an effective neuroprotectant against early brain injury and learning deficits induced by subarachnoid hemorrhage: possible involvement of inflammatory signaling. Journal of Neuroinflammation, 10(1), 157. http://dx.doi.org/10.1186/1742-2094-10-157. PMid:24373431.

Tu, E., Chen, Q., Tan, L., \& Wang, Y. (2020). Dl-3-n-Butylphthalide promotes neovascularization and neurological recovery in a rat 
model of intracerebral hemorrhage. BMC Neuroscience, 21(1), 24. http://dx.doi.org/10.1186/s12868-020-00575-3. PMid:32471341.

Venna, V. R., Li, J., Benashski, S. E., Tarabishy, S., \& McCullough, L. D. (2012). Preconditioning induces sustained neuroprotection by downregulation of adenosine 5 '-monophosphate-activated protein kinase. Neuroscience, 201, 280-287. http://dx.doi.org/10.1016/j. neuroscience.2011.11.014. PMid:22120436.

Wang, K. C., Tang, S. C., Lee, J.E., Tsai, J. C., Lai, D. M., Lin, W. C., Lin, C. P., Tu, Y. K., \& Hsieh, S. T. (2018). Impaired microcirculation after subarachnoid hemorrhage in an in vivo animal model. Scientific Reports, 8(1), 13315. http://dx.doi.org/10.1038/s41598-018-31709-7. PMid:30190518.

Wang, M., Frasch, S. C., Li, G., Feng, D., Gao, B., Xu, L., Ir, D., Frank, D. N., Bratton, D. L., \& Ju, C. (2017). Role of gp91(phox) in hepatic macrophage programming and alcoholic liver disease. Hepatol Commun, 1(8), 765-779. http://dx.doi.org/10.1002/hep4.1078. PMid:29404493.
Ye, Z. Y., Xing, H. Y., Wang, B., Liu, M., \& Lv, P. Y. (2019). DL-3-nbutylphthalide protects the blood-brain barrier against ischemia/ hypoxia injury via upregulation of tight junction proteins. Chinese Medical Journal, 132(11), 1344-1353. http://dx.doi.org/10.1097/ CM9.0000000000000232. PMid:30939485.

Zhu, Q., Enkhjargal, B., Huang, L., Zhang, T., Sun, C., Xie, Z., Wu, P., Mo, J., Tang, J., Xie, Z., \& Zhang, J. H. (2018). Aggf1 attenuates neuroinflammation and $\mathrm{BBB}$ disruption via PI3K/Akt/NF- $\kappa \mathrm{B}$ pathway after subarachnoid hemorrhage in rats. Journal of Neuroinflammation, 15(1), 178. http://dx.doi.org/10.1186/s12974018-1211-8. PMid:29885663.

Zolnourian, A., Galea, I., \& Bulters, D. (2019). Neuroprotective role of the Nrf2 pathway in subarachnoid haemorrhage and its therapeutic potential. Oxidative Medicine and Cellular Longevity, 2019, 6218239. http://dx.doi.org/10.1155/2019/6218239. PMid:31191800. 